\title{
Complexity Problems Handled by Advanced Computer Simulation Technology in Smart Cities 2021
}

\author{
Zhihan Lv (D), ${ }^{1}$ Kaoru Ota, ${ }^{2}$ Jaime Lloret $\mathbb{D},{ }^{3}$ Wei Xiang, ${ }^{4}$ and Paolo Bellavista ${ }^{5}{ }^{5}$ \\ ${ }^{1}$ Qingdao University, Qingdao, China \\ ${ }^{2}$ Muroran Institute of Technology, Muroran, Japan \\ ${ }^{3}$ Instituto de Investigacion para la Gestion Integrada de Zonas Costeras, Universitat Politecnica de Valencia, Spain \\ ${ }^{4}$ James Cook University, Cairns, Australia \\ ${ }^{5}$ Università di Bologna, Bologna, Italy \\ Correspondence should be addressed to Zhihan Lv; lvzhihan@gmail.com
}

Received 5 January 2022; Accepted 5 January 2022; Published 2 February 2022

Copyright (c) 2022 Zhihan Lv et al. This is an open access article distributed under the Creative Commons Attribution License, which permits unrestricted use, distribution, and reproduction in any medium, provided the original work is properly cited.

Complexity science technology will bring opportunities for the continuous expansion of new simulation research fields, especially for human simulation, social simulation, and human brain simulation, and provide unprecedented opportunities for simulation for social governance, prediction, and urbanization. The development of complexity science technology provides an important opportunity to use modeling and simulation methods to study new virtual information spaces. As we all know, the advantage of modeling and simulation technology is to provide a bridge from the real world to the virtual space, which provides a substitute for studying the unknown fields of human society and the physical world.

However, virtual information spaces are a completely new virtual space that are completely different from the physical world and human society. This virtual space is not only "human, machine, and object" but also a space where the three notions are combined, and there are also the characteristics of multilayer mesh and cross-domain association. The study of this new virtual space is a complex and current scientific research area. On the basis of making full use of the results of complexity science research, using parallel systems, embedded simulation, and other methods to build a bridge to this new type of space, they provide a virtual alternative for the interaction or behavioral characteristics between the research. Using the results of complexity science to constantly modify and test the models built is an issue that needs to be addressed for the further development of modeling and simulation science.
This special issue collates 189 original research papers and comments with a focus on up-to-date technology related to computer simulation for complexity issues in smart cities and serves as a forum for researchers all over the world to discuss their work and recent advances in this field. In particular, this special issue aims to showcase the most recent achievements and developments in complexity problem discovery and exploration.

With the development of science and technology, the demand of human-computer interaction has increased, and the research on human-computer interaction in real and virtual scenarios has become more and more extensive. The use of virtual reality technology and the combination of human-computer interaction technology have been increasingly used in the fields of simulated sports, medical rehabilitation, and game creation. Action is the basis of human behaviours, among which human behaviours and movement analysis are an important research direction. Among human behaviours and actions, the recognition research based on behaviours and actions has the characteristics of convenience, intuition, strong interaction, and rich expression information. It has become the first choice for many researchers to analyse human behaviours. At the same time, the study of some complex objects such as human movement has many fuzzy factors, which are difficult to express and deal with. A total of 26 research papers in this special issue propose processing methods and optimization algorithms for human-computer interaction applications in multiple scenarios, providing solutions to complex human- 
computer interaction problems from many aspects, and all have good application performance.

Urban digital twins are virtual representations of urban physical assets and are an important development direction of digital twin technology. Smart cities built under the support of digital twin technology can bring better cost efficiencies, operational efficiencies, better crisis management, more openness and better-informed decision-making, more participatory governance, or better urban planning. The special issue contains 5 representative research papers on smart city construction. I. Meta et al. proposed a modular framework for urban digital twins, combined with the concept of urban physiology, to better solve the theoretical problems of smart city construction. Y. He et al. proposed a knowledge graph fusion framework for building smart cities. S. Wang et al. provided a feature extraction method for the spatial distribution of urban landforms. Starting from the energy efficiency management of smart cities, L. Chen and P. Han constructed an efficient system platform based on the mobile data of the Internet of Things. H. Wang et al. improved the KNN algorithm and optimized the preprocessing process of the city center. In addition, the special issue also includes a total of 129 papers on the optimization methods of urban life in other fields, which include research methods from different perspectives of urban development and urban life, which optimize and enrich urban construction and daily need to a great extent. These areas include education, healthcare, construction, tourism development, financial transactions, entertainment consumption, urban security services, low-carbon and green development, urban resource allocation and control optimization, environmental and geological monitoring, urban production, sports, and urban transportation. In these areas, this special issue brings together a wealth of optimization methods.

In the field of education, the complexity of the education system at all levels has gradually deepened. Whether it is a junior high school, high school, or university, excellent student management systems, online education systems, teaching quality evaluation, and more intelligent teaching models are the main development directions. The special issue contains 32 education-related papers. Starting from the practical development of subject education in many aspects, a series of optimization algorithms and platform construction methods are proposed. In addition, this is not only limited to conventional teaching types but also includes painting, sports, and student psychological supervision, which promote the intelligent development of education in a more comprehensive manner.

In terms of healthcare, Y. Chen proposed an optimized plan for the clinical nursing management system to make the overall clinical nursing process more perfect. Y. Jiang et al. also proposed an optimized method for analyzing clinical information. Aiming at the fusion of supporting medical image data on the ground, $\mathrm{R}$. Chen et al. proposed a system framework that breaks through some of the limitations of traditional image fusion. X. Che compared the difference between exercise intervention and other nondrug therapies. B. Yang and S. Wang investigated the cognitive function.
For the field of urban building structure, here are some further improvements based on typical treatment methods. J. Yu et al. put forward a method on how to achieve healthy development of contaminated areas and facility construction. M. Wei combined augmented reality to realize more intuitive visualization of building data and intelligent detection. Other papers include such as the treatment method of building fire protection, the distribution method of housing construction in some newly developed areas, the virtual reality construction technology of landscape architecture, the intelligent combination of architecture and environment, and landscape space layout or landscape planning and design method. The special issue contains 4 papers on tourism. With the development of the national economy and society, the scale of the tourism market has expanded rapidly. The informatization and intelligent construction of scenic spots cannot keep up with the pace of economic development, and there is a lack of effective management methods to predict or even improve the overloading of scenic spots, which objectively leads to problems of congestion and overload. More and more tourists flock to the scenic area, which brings greater pressure and safety hazards to the scenic area. The relevant research in this part effectively responded to this problem, formulated a suitable flow control plan, and promoted the intelligent development of scenic spot management.

In the field of urban economy, it includes financial transactions, entertainment consumption, and urban security services. The problem of economic structure equilibrium and its development trend have far-reaching theoretical significance and important practical value. In theory, the study of economic structure equilibrium is an intuitive reflection and detailed elaboration of the status quo of economic development and is an extension and expansion of economic research theories. The changes in the economic structure are more complicated, and the problem of building intelligent economic industry forecasting models needs to be solved urgently. It is very important to study the balance of economic structure and economic development trends and to carry out macrocontrol on the status and trends of economic development. This part contains 39 research papers and 1 comment. In terms of entertainment consumption, the special issue includes movie recommendation, movie big data analysis, and other movierelated applications. At the same time, there are many papers about music feature optimization, music signal segmentation retrieval, and other applications. In urban security, social security services, logistics services, and effective optimization of news reports promote the lives of residents and social security from multiple perspectives. By combining advanced technologies such as big data, Internet of Things, and artificial intelligence, it has promoted the intelligent development of urban economy and entertainment guarantee.

A total of 20 papers were included to discuss the lowcarbon and green development of the city, the allocation of urban resources, and the optimization of control. Against the background of the development of innovative countries, climate change and environmental issues have become increasingly prominent. Low-carbon economy has become an important choice for the future economic development of all 
countries, and the green development of cities is an inevitable trend. Representatives such as the twin cities proposed by Y. Liu et al. provide a new perspective for the development of urban intelligence. $\mathrm{H}$. Liu integrated low-carbon concepts into urban development. Xu et al. proposed digital technology to help food supply and provided corresponding optimization solutions. L. Zhang built a marketing management system based on cloud computing and big data. S. Mao and R. Huang also proposed intelligent construction methods for marketing strategies. The development of emerging technologies improves the basic appearance of cities by regulating the rational allocation of urban resources. Urban services also include research on sports, urban production, basic transportation, and environmental monitoring. Among them, there are 9 sports-related papers, and most of the papers contain related concepts of humancomputer interaction, which can realize the monitoring and modeling of complex movements in some sports, and make a certain contribution to the intelligent development of urban sports. In terms of environmental monitoring, J. Jin et al. provided supporting technologies for geological monitoring in important strategic areas in some countries.

Some new data processing methods in the background of big data are also proposed here. X. Wang et al. proposed a digital image processing method based on the geographic information system. X. Xu et al. combined virtual reality technology to realize the restoration of similar images. S. Wu et al. used virtual reconstruction technology to acquire 3D images. X. Liu created a 3D modeling method that is more in line with image sequences. In addition, the special issue includes 21 algorithm optimization methods, covering most fields such as deep learning, blockchain distributed computing, edge computing, animation synthesis, and semantic similarity algorithms, providing many algorithm optimizations for different field methods.

This special issue has made important contributions to all the fields mentioned above and has promoted the development of the complexity science of smart cities from multiple perspectives. Combining the new generation of information technology and big data technology in the Innovation 2.0 environment, it strengthens open data and social participation in smart cities, solves the problems of open data and privacy protection in the context of big data, and lays the foundation for the construction of new smart cities.

\section{Conflicts of Interest}

The editors declare that there are no conflicts of interest regarding the publication of this paper.

Zhihan Lv

Kaoru Ota

Jaime Lloret

Wei Xiang

Paolo Bellavista 